\title{
The Practice of Argumentative Discussion
}

\author{
DAVID HITCHCOCK \\ Department of Philosophy \\ McMaster University \\ Hamilton, Ontario \\ Canada L8S 4K1 \\ E-mail: hitchckd@mcmaster.ca
}




\title{
The practice of argumentative discussion
}

\author{
ABSTRACT. I propose some changes to the conceptions of argument and of argumentative \\ discussion in Ralph Johnson's Manifest Rationality (2000). An argument is a discourse \\ whose author seeks to persuade an audience to accept a thesis by producing reasons in \\ support of it and discharging his dialectical obligations. An argumentative discussion (what \\ Johnson calls 'argumentation') is a socioc ultural activity of constructing, presenting, \\ interpreting, criticizing, and revising arguments for the purpose of reaching a shared \\ rationally supported position on some issue. Johnson's theory of argumentative discussion, \\ with occasional modifications, is derived from this definition as a sequence of 17 theorems. \\ Argumentative discussion is a valuable cultural practice; it is the most secure route to \\ correct views and wise policies.
}

KEY WORDS: Ralph Johnson, argument, argumentation, argumentative discussion, practice, definition, manifest rationality, definition, communication, rhetoric

In his Manifest Rationality, Ralph Johnson (2000) singles out for attention a practice he calls argumentation, 'the sociocultural activity of constructing, presenting, interpreting, criticizing, and revising arguments.' $\left(12,154^{1}\right)$ I propose to reconstruct the structure of Johnson's thinking about this practice, to give reason s for generally endorsing that thinking, to propose some alterations to it, and to explain why the practice is valuable. 


\section{SOME SPECIES OF ARGUMENTATION}

The definition quoted above presupposes that there is exactly one sociocultural activity, perhaps occurring at many times and places, which incorporates the dimensions mentioned in a single whole. It is a socially established cooperative activity with internal standards of excellence (155), analogous in this respect to the practice of exchanging gifts on some festive occasion. The activities of those engaging in it receive their intelligibility within the practice (155), but the practice as a whole needs to be understood within the customs, habits and activities of the broader society (154).

Is there a practice of the kind Johnson defines? Perhaps surprisingly, he cites no examples. He does cite some (alleged) products of the practice: an argument against a novelist's characterization of a city as grimy (33), a defence of a university president's refusal to fire a controversial professor (33-34), and Immanuel Kant's Critique of Pure Reason, construed as an argument that the faculty of reason is limited and cannot prove metaphysical theses (35). The interchanges in which these arguments are embedded, however, do not at first glance exhibit the features of one person interpreting and criticizing an argument and the argument's author revising it in response to this criticism, features which are constitutive of the practice of argumentation as Johnson defines it. We are given some examples of issues which may be addressed by arguments deployed within the practice of argumentation: alcoholism (303), litter (308), cigarette smoking (308), casinos (309), but no examples of arguments on such issues embedded in the kind of practice presupposed by Johnson's definition. 
I therefore cite six species of the practice of argumentation as he defines it. First, people express their opinion about some issue and support it by reasons in letters to newspaper editors, radio phone-in shows, Internet discussion groups, panel discussions on television shows and so on; they interpret others' arguments, criticize them, and modify their own arguments in the light of criticism. Second, referees of journal submissions and book manuscripts interpret authors' arguments, criticize them and propose revisions which the authors either reject with reasons or accept. Third, scholars and scientists discuss in publications and at conferences such issues as the health effects of tobacco smoking or the cause of AIDS or whether reasons are causes. Fourth, judges support their opinions with reasons, reasons which take into account the arguments advanced by attorneys on either side of a given case and in the case of dissenting opinions the opinions and arguments of the majority of the court. Fifth, families and friends discuss issues informally in back-andforth exchanges. Sixth, at meetings groups of various kinds discuss issues before they make a decision.

These practices share the features of argumentation as Johnson defines it: a participant will construct and put forth an argument, another participant will interpret and criticize it, and the first participant will respond, perhaps by revising the argument. Each practice has internal criteria of intelligibility and internal standards of excellence which are similar enough that they can be regarded as species of a single well-defined genus. Thus the aforementioned presupposition of Johnson's definition has been established. 
The Practice of Argumentative Discussion

\section{THE DEFINITION OF ARGUMENT}

A second presupposition of Johnson's definition is that we can identify what an argument is independently of recognizing instances of the practice of argumentation; otherwise the definition would be objectionably circular. Johnson himself offers the following canonical definition of argument:

An argument is a type of discourse or text-the distillate of the practice of argumentation-in which the arguer seeks to persuade the Other(s) of the truth of a thesis by producing the reasons that support it. In addition to this illative core, an argument possesses a dialectical tier in which the arguer discharges his dialectical obligations. (168)

This definition needs some changes to be acceptable. To avoid objectionable circularity, we need to remove the reference to the practice of argumentation. To accommodate arguments for courses of action (e.g. voting for candidate X or party Y), we should characterize the arguer's goal as securing acceptance of a thesis rather than recognition of the truth of a thesis; the predicates of truth and falsity simply do not apply to many theses for which people argue. We need to remove the (probably unintended) suggestions that an argument must include all the reasons which support the claim argued for and must include only reasons which actually support it. Finally, to make the definition intelligible in abstraction from its context, we need to spell out what is meant by 'the Other(s).' These changes collectively generate the following revised definition:

An argument is a spoken discourse or written text whose author (the arguer) seeks 
to persuade an intended audience or readership (the Other or the Others) to accept a thesis by producing reasons in support of it. In addition to this illative core, an argument possesses a dialectical tier in which the arguer discharges his dialectical obligations.

Dialectical obligations include responses to objections and consideration of alternative positions.

Johnson's definition has the consequence that a person does not produce an argument who merely adduces reasons in support of a thesis, but does not discharge her dialectical

obligations. Such products often emerge from the six types of argumentation I listed above. Johnson calls them 'proto-arguments' (170); they lack a structural component which a full argument has.

\section{THE DEFINITION OF ARGUMENTATION}

Removing from the definition of argument any reference to the practice of argumentation, in order to avoid circularity in the definition of the practice, has the consequence that arguments occur in other contexts than argumentation. In their summary arguments, for example, lawyers for the prosecution or plaintiff and for the defendant produce reasons in support of a central thesis (that the defendant should be found guilty as charged or held liable, or not) and may respond to objections. So such lawyers produce arguments. But the act of advancing such arguments in an attempt to convince a judge or jury is not part of a practice of argumentation, according to Johnson (153), because it does not obey the 
fundamental principle internal to this practice that the strength of the better reason and nothing else shall determine its outcome. In the courtroom, 'the skills of the barrister, the rhetorical presence, the strategy used in selecting the jury, cross-examination skills, a superior information base, the advocate's shrewdness ... may play a greater role in determining the outcome than the force of the advocate's reasoning.' (153) Similarly, arguments in rhetorical contexts where the goal is effective persuasion are not part of a practice of argumentation, because the goal of effective persuasion can often be met better by ignoring objections (270).

I believe that Johnson is correct to distinguish the interventions of lawyers in a legal proceeding or of partisan participants in debate from the practice of argumentation to which he draws our attention. The difference is however not captured by his proposed definition. Opposing lawyers or candidates or spokespersons may quite easily construct and present arguments, interpret and criticize their opponents' arguments, and revise their arguments in response to criticism. But in such rhetorically driven exchanges competing participants aim to secure or intensify adherence of an audience to their favoured thesis, whereas participants in argumentation are (or are supposed to be) aiming at a rationally supported position on an issue. To distinguish practices whose participants are (and are recognized as being) rhetorically driven from those whose participants are (or are supposed to be) rationally driven, we must supplement Johnson's definition.

In doing so, we should aim to characterize the purpose of the participants in a genuine argumentation, rather than the function of the practice. The (at least pretended) purposes of 
participants in a communicative practice can fairly easily be established from their typical behaviour and from culturally accepted norms whose violation attracts disapproval. The function of the practice, on the other hand, is not always evident; its discovery requires investigation, and so (on pain of vicious circularity) it cannot be part of the definition which we use to determine whether a given communicative interaction is an instance of the practice. Admittedly, Johnson talks about 'the fundamental purpose' of the practice of argumentation, and characterizes it variously as 'to arrive at the truth about some issue' (158) and 'rational persuasion' (159). Despite appearances, however, these characterizations describe the expected purpose of the participants rather than the function of the practice. That purpose might better be described as reaching a shared rationally supported position on some issue, because argumentation can be about what to do as well as about what is the case, and because argumentation focuses on the opinions of its participants, not on those of a non-participating audience (as trials, for example, and election campaigns do).

Further, to characterize the telos of argumentation as rational persuasion is to embrace a rhetorical conception of its function; rhetoric, in Aristotle's classic definition, is 'an ability to observe in each case the possible means of persuasion.' (Ars Rhetorica I.2.1355b25-26) A rhetorical conception of the telos of argumentation makes it hard to argue for the obligation of an arguer to respond to objections; from a rhetorical perspective, it may be perfectly rational to ignore certain objections. Even construing the goal as rational persuasion may not rule out appeals to ethos and pathos in the way that Johnson 
wishes to, since these can play an ancillary role without making persuasion irrational.

I therefore propose as the overall expected purpose of participants in the practice of argumentation that of arriving at a shared rationally supported position on an issue. The reference to rational support makes sense of the consideration of objections, openness to criticism and willingness to revise arguments which Johnson rightly cherishes as hallmarks of the practice. It explains how the goals which Johnson regards as internal to it (increase in rationality of the participants and being rationally persuaded or coming closer to an acceptable position [155]) in fact follow from its definition.

I would like also to propose a change of name. The word argumentation is already in common use for what Johnson characterizes as the 'illative core' of argument, the offering of reasons in support of a position. The 'argumentation schemes' to which he refers are schemes for argumentation in this sense. Van Eemeren and Grootendorst's $(1984,1992)$ characterize what they call 'argumentation' in this sense as a complex speech act; the 'argumentation stage' of their four-stage normative model of an argumentative discussion is the stage in which arguments are presented. I propose therefore that we call the practice to which Johnson draws our attention argumentative discussion, the designation it receives in the title of van Eemeren and Grootendorst's (1984). Then our revised definition would read as follows:

An argumentative discussion is a sociocultural activity of constructing, presenting, interpreting, criticizing, and revising arguments for the purpose of reaching a shared rationally supported position on some issue. 


\section{APPARENT COUNTER-EXAMPLES}

This definition, it might be objected, attributes to participants in argumentative discussions a purity of intention which real-life participants in the six practices described earlier often do not possess. People who write letters to the editor, phone radio talk shows, send messages to Internet discussion groups and join in panel discussions are often more concerned to assert their own position than to engage in a back-and-forth interchange which might lead them to change their mind. Referees may push their own view rather than suggest ways of strengthening the argument in a manuscript. Scholars and scientists may cling stubbornly to a favoured view long after it has been decisively refuted. Similarly for judicial and quasi-judicial opinions, discussions among family and friends, and decisionfocussed discussions at meetings.

There are two possible responses to such apparent counter-examples. One is to weaken the definition of argumentative discussion so as to embrace the counter-examples; one might for example speak about the ostensible purpose of the participants. Then one would develop norms for the good conduct of argumentative discussions; ego-involved or partisan participants would be said to be engaging in an argumentative discussion, but doing so in bad faith. The other response is to stick to the definition and deny that the apparent counterexamples are really counter-examples. Ego-involved or partisan contributors to a spoken or written exchange may pretend to be involved in an argumentative discussion, but they are really not discussing. They are debating. Thus the normative criteria for good argumentative discussion are built right into its definition. Any argumentative discussion is by definition a 
good argumentative discussion (according to the internal standards of the practice). There is no such thing as a bad argumentative discussion. If it is a bad form of communication according to the standards of the practice of argumentative discussion, then it is not really argumentative discussion, whatever the pers on engaged in the communication may say.

These two responses exemplify two possible strategies for making a concept precise. The descriptive strategy, exemplified by broadening the proposed definition of argumentative discussion, is to start with a broad descriptive characterization (e.g. of art, music, philosophy, critical thinking) and then develop evaluative criteria for distinguishing better instances from worse ones. (On the negative side, one would start with a broad descriptive characterization of a concept like suicide or murder or ad hominem attack, and then distinguish within the broad class covered by this characterization between justifiable and unjustifiable instances.) The prescriptive strategy is to start with an honorific (or pejorative) characterization with the norms built in and use some qualified label for instances which fail to meet the norms. Is one of these strategies objectively more correct than the other? Are the fluorescent paintings on black velvet backgrounds which are sold in the parking lots of neighbourhood strip malls bad art or pseudo-art? Is a defence lawyer's exposure of the personal failings of a key witness an ad hominem attack justified by its relevance to impugning the credibility of the witness's testimony, or not an ad hominem attack at all, since the lawyer commits no fallacy? Is a grossly partisan participant in a panel discussion on some political question a bad discussant, or someone who is not really discussing at all? There seems to be no fact of the matter about the answers to these 
questions; it all depends on how one uses the key word in question. Both the descriptive and the prescriptive strategies have their point. A descriptive strategy makes it easier to identify cases which fall under the concept; one does not need to check for conformity to norms (positive or negative) in order to call something art, argumentative discussion, music, philosophy, critical thinking, suicide, murder, ad hominem attack, or whatever. On the other hand, extending the concept to cases outside the usual norms debases the honorific or condemnatory force in the ordinary usage of the term; if some murders are excusable, or even justifiable, how are we to preserve the outrage associated with calling something an act of murder? The pres criptive strategy preserves the normative force of our terminology, but at the price of making it a more complicated task to discover whether a term applies to a given case. Each strategy's strength is the other one's weakness.

The decision between the two strategies is not a mere matter of stipulation. The acceptability of Johnson's definition and of the norms incorporated in it depends on the existence of a certain practice and the recognition within it of the norms in his definition. The practice of argumentative discussion does seem to exist, in our culture and in others; its participants seem to recognize as a norm the goal of arriving at a shared rationally supported position on an issue. So Johnson's definition is defensible, and the prescriptive strategy for dealing with apparent counter-examples is defensible.

\section{PROPERTIES OF ARGUMENTATIVE DISCUSSION}

The modified definition of argumentative discussion distinguishes it from similar but more 
rhetorically driven practices and thus makes possible the derivation of the characteristics Johnson attributes to argumentative discussions as a theory of argumentative discussion. Unlike mathematical proofs, the following derivations depend on implicit assumptions and involve non-conclusive inferences, whose conclusions are vulnerable to undermining or overriding defeaters. Thus these 'proofs' are subject to the sort of criticism and future revision which Johnson regards as central to an argumentative discussion.

In some cases, I have found it necessary to revise some of Johnson's 'theorems'. I note such revisions in the comment section following the 'proof'.

Theorem 1: A participant in argumentative discussion who makes a claim which requires rational support must support it with reasons. Proof: If the participants are to arrive at a shared rationally supported position on the issue under discussion, then their acceptance of any claim as a basis for their shared position must be rea sonable. A claim made by a participant in an argumentative discussion is a contribution to arriving at a shared rationally supported position. Hence either it is reasonable for the other participants to accept this claim without rational support or the maker of the claim must support it by reasons.

Comment: Johnson asserts that a participant in an argumentative discussion who makes any claim must support it with reasons. $(158,160,162)$ This assertion is subject to an infinite regress, if we assume that each reason is itself a claim and thus needs to be supported by reasons. Johnson's remark that 'participants in the practice [of argumentative discussion-DH] recognize that any claim made must be supported by reasons or evidence' (160) allows one way out of the regress-producing physical evidence, for example pointing 
to ominous dark clouds to the west in support of one's claim that it will be raining soon.

But we ought to allow other ways; eye-witness testimony, expert opinion and shared reasonable assumptions, for example, generally do not require supporting reasons. Thus not every failure to support a claim with reasons means that conclusions drawn from that claim lack rational support. The qualification 'which requires rational support' points up the need for a theory of rational support.

Theorem 2: Participants in an argumentative discussion may appeal to reasons (including physical evidence) and only to reasons. (311) Proof: If one appeals to anything other than reasons or evidence in order to get a claim accepted (e.g. presenting oneself as a person of admirable character whose word is to be accepted just on one's say-so, using intimidating tactics, stirring up emotions in a way which is not justified by the substance of one's argument), then those who accept the claim on this basis will not have rational support for it. Thus the aim of reaching an agreed rationally supported position will be undermined. Comment: 'Reasons' are taken to include evidence in order to accommodate Johnson's allowance of evidence as a rational starting-point for an argument.

Theorem 3: A participant may not resort to trickery or force to get a claim accepted. (319) Proof: A claim accepted even partly on the basis of trickery or force is not accepted because of rational support. Therefore, it and any conclusions drawn from it are not assured of being rationally supported.

Theorem 4: An argumentative discussion presupposes a background of controversy (or potential controversy) about the issue under discussion. (160) Proof: If there were not even 
potential controversy about the issue, then there would be no need to construct, present, interpret, criticize and revise arguments in order to arrive at an agreed rationally supported position on the issue. If some or all the participants already had a common rationally supported position on the issue, and any without such a position were merely perplexed, it would be enough for one person to state the position with its rational support. If all participants are perplexed, but an exploration of facts relevant to settling the issue has no potential to lead to objections and criticisms (because for example a rationally well supported algorithm applies), then argumentative discussion is inappropriate. Comment: Johnson requires actual controversy about an issue as a presupposition of argumentative discussion. But argumentative discussion seems appropriate also in cases of perplexity where no controversy yet exists, but investigation will likely produce it-e.g. the case of identifying the cause of Acquired Immune Deficiency Syndrome (AIDS) when the syndrome was first identified, before anyone had opinions about its etiology.

Theorem 5: The author of an argument in an argumentative discussion has a responsibility to deal with known alternative positions and with known objections. (165, 318) Proof: Since the issue in a argumentative discussion is controversial or potentially controversial (theorem 4), the Others whom the arguer wishes to convert to the arguer's position may know of alternative positions and objections, and the arguer will know that they may know them. To ignore them would be to fail to engage in the process of arriving at a shared rationally supported position. (160) To the extent that the arguer does not consider a known alternative position or deal with a known objection, the conclusion is less fully 
rationally supported than it otherwise would be. If rational consideration of an ignored objection would require modification of the conclusion, for example, then the conclusion is not rationally supported. If there is a rational response to the ignored objection consistent with the conclusion, then the audience (and perhaps the arguer) is deprived of the additional rational support derived from knowing that a known objection can be defused. Similarly for known alternative positions.

Theorem 6: The addressees of an argument in an argumentative discussion have a responsibility to provide criticism of that argument if they believe it warranted, and the arguer has a responsibility to welcome and deal with their criticisms. The arguer agrees to let feedback from the Other affect the product, to take criticism seriously; intervention of the Other is not just accepted, but is encouraged, so as to make the product better.(158, 161) Proof: If the criticism is in fact justified, then addressing it to the arguer increases the chance that the argument will be modified so as to be more rationally supported, and thus increases the chance that the ultimate outcome of the discussion will be an agreed rationally supported position. If the criticism is not justified, then addressing it to the arguer increases the chance that its weakness will be made evident to the author of the criticism, thus dispelling an unjustified mental reservation about the argument and so strengthening adherence to a rationally supported claim. Comment: This feature of argumentative discussion makes puzzling Johnson's privileging of written over oral argumentative discussions. While it is true that written arguments are more stable-and thus more amenable to analysis, evaluation and criticism-oral discussions lend themselves much more 
easily to fluid exchanges of tentative formulations open to revision on the fly. Wohlrapp's work on retroflexive argumentation (Wohlrapp 1998) and Willard's work on argumentation as dissensus (Willard 1983, 1989) are good examples of theorizing forms of argumentative discussion which are much less stilted and fixed than written exchanges.

Theorem 7: Contributions to an argumentative discussion must not only be rational, but must be seen by the participants to be rational. $(144,163,317-318)$ Proof: We assume that participants in an argumentative discussion aim not just to agree on a rationally supported position, but to agree on a position because they know it is rationally supported. Fulfilment of this goal requires that they recognize the rationality of each contribution. This requires that the contribution be made in such a way as to secure this recognition. Comment: The requirement that contributions to an argumentative discussion be seen by its participants to be rational is the requirement of manifest rationality which gives Johnson's book its title.

Theorem 8: The outcome of an argumentative discussion is to be determined only by the strength of the better reason. $(153,160)$ Proof: If anything else even partially determines the outcome, then there is no assurance that the better reason actually favours the outcome reached and thus no assurance that it is rationally supported.

Theorem 9: Participants in an argumentative discussion must be rational, and must know that they are rational. $(162,164$ n. 15) Proof: Construe rationality in terms of a disposition to support claims by reasons or evidence when they need such support, to acknowledge the force of reasons, and so forth. To be rational in this sense is a requirement of arriving through discussion at an agreed rationally supported position. If the participants 
are not just to agree on a position which is in fact rationally supported, but are to recognize that it is rationally supported, then they must each have the rationality required to recognize rational support and they must recognize that each of them has it.

Theorem 10: The participants in an argumentative discussion embrace, endorse and cherish rationality. $(12,14,161,162)$ Proof: Rationality consists in giving and receiving reasons (14) or in using, giving or acting on reasons (161). Willing participants in a discussion which is devoted solely to giving, receiving, responding to and modifying reasons obviously cherish doing so. Comment: Since a tricky argument nonetheless presents a reason supporting a position, and a threat is a reason for bowing to it, we need a further specification of rationality than 'giving and receiving reasons' to rule out trickery and threats as non-rational or even irrational.

Theorem 11: An internal good of argumentative discussions is an increase in rationality among the participants and thus an increase in rationality in the world. Specifically, participants acquire a deeper understanding of the issue, or are rationally persuaded of a certain position on it, or come closer to an acceptable position. $(155,162)$ Proof: An arguer who sees and accepts a critic's objection has a more rational position on the issue addressed in the argument, whereas an arguer who shows that a critic's objection is wanting has a more rational position in virtue of having warded off objections. (162) The audience too, including the critic, has a more rational position as a result of either of these outcomes. The participants exhibit rationality by giving reasons, weighing objections, modifying positions to accommodate them; the arguer acknowledges the critic's objections, the critic 
acknowledges rationality in the arguer's position. (162-163) Comment: One might object that there are other possible sequences than argument, criticism, and acceptance or refutation of criticism. The arguer might just reject a critic's objection without having found it wanting. Or the arguer might accept an objection when there is a good reason for rejecting it. Or the potential critic might fail to bring forward a crucial objection, and instead accept the argument, thus reinforcing the arguer (and the critic) in an irrational commitment to the arguer's position. Or the discussion might degenerate into angry personally abusive recriminations. These outcomes however would transform the communicative interchange into something less than an argumentative discussion. It would have degenerated into egocentric dismissal of justified criticism, lazy acceptance of an interlocutor's statement without careful scrutiny, 'polite' acquiescence despite awareness of an objection, or appeal to something other than the strength of the reasons.

Theorem 12: Argumentative discussion depends on a specifically human form of rationality. (12) Proof: Other animals are not able to give and receive reasons. (13) Corollary: Only humans engage in argumentative discussion. (162)

Theorem 13: Argumentative discussion is not the only rational process. Proof: Proving and theorizing are distinct rational processes. (162) Comment: Though distinct from argumentative discussion, proving shares the characteristic of manifest rationality. The point of a proof is to show to an intended audience that a conclusion follows necessarily from given assumptions. Hence at each step the intended audience must be able to see how the conclusion is drawn. The proof must be seen to be rational. 
Theorem 14: A culture has a practice of argumentative discussion only if its members have a common interest in inquiry, getting at the truth or persuasion. $(15,16)$ Proof: By definition, the practice requires its participants to aim to reach a shared rationally supported position on some issue. Hence the participants in an argumentative discussion must have a common interest in achieving this goal. Comment: Not all members of a culture need share this interest, nor need they share it on all issues. It is enough if there are groups which communicate internally and share this interest on some issues. Johnson's apparent assumption to the contrary (16) seems overly strong.

Theorem 15: A culture has a practice of argumentative discussion only if its members seriously disagree about some important issues (as opposed for example to subscribing jointly to some mythopoetic standpoint). (15) Proof: From theorem 4.

Theorem 16: A culture has a practice of argumentative discussion only if its members understand and value rationality as a means of achieving their common interest. (15) Proof: If members of a culture did not value rationality as a means of achieving their common interest in truth or persuasion, then they would not participate in and endorse a practice which requires its participants to appeal only to the force of the better reason in reaching agreement on a controversial issue. Comment: This valuing of rationality need not be universal, either among the members of the culture or with respect to all issues.

Theorem 17: A culture has a practice of argumentative discussion only if its members are open to changing their view as a result of argument. (15) Proof: From theorem 6. 


\section{THE VALUE AND PRESENT STATUS OF ARGUMENTATIVE DISCUSSION}

Johnson believes that argumentative discussion is 'an extremely powerful and valuable cultural practice' (11). It might not be amiss to say why. It is powerful because it has the potential, like few other practices, to change ignorance into knowledge and prejudice into reasoned judgement. The controversial issues which are the focus of argumentative discussion include issues about far-reaching questions of public policy. By changing opinion about those issues, argumentative discussion can thus have an immense impact on human lives.

This impact is generally an impact for good. Human well-being (and the well-being of animals, species, the biosphere and our planet) is served best by positions and policies which reason would support. While intuition or precedent or ideology might arrive at a correct position and a wise policy, such methods are chancy at best, dangerous at worst. (Where they are reliable, of course, rea son will en dorse them.) Partisan debate, al though sharing some features of rationality with argumentative discussion, is strongly affected by non-rational or even irrational influences. Free and open rational discussion, welcoming criticism and willing to change in the light of that criticism, is the most secure route to correct views and wise policies.

\section{NOTES}

${ }^{1}$ Page references here and in what follows are to Johnson (2000). Johnson omits the word criticizing in the second place cited. 


\section{REFERENCES}

Aristotle: 1959, Ars Rhetorica, Clarendon Press, Oxford. (First published ca. 350 BCE.)

Eemeren, Frans H. van, and Rob Grootendorst: 1984, Speech Acts in Argumentative Discussions, Foris, Dordrecht.

Eemeren, Frans H. van, and Rob Grootendorst: 1992, Argumentation, Communication and Fallacies, Lawrence Erlbaum Associates, Mahwah, NJ.

Johnson, Ralph H.: 2000, Manifest Rationality: A Pragmatic Theory of Argument, Lawrence Erlbaum Associates, Mahwah, NJ.

Willard, Charles A.: 1983, Argumentation and the Social Grounds of Knowledge, University of Alabama Press, Tuscaloosa.

Willard, Charles A.: 1989, A Theory of Argumentation, University of Alabama Press, Tuscaloosa.

Wohlrapp, Harald: 1998, 'A new light on non-deductive argumentation schemes', Argumentation 12, 341-350. 